\title{
ADAPTACIÓN Y VALIDACIÓN PRELIMINAR DE LA FORMA ESPAÑOLA DE LA ESCALA CAMI-S (COMMUNITY ATTITUDES TOWARDS MENTAL ILLNESS)
}

\section{MERITXELL SASTRE-RUS ${ }^{1}$, JAVIER MONTES-HIDALGO², M. ${ }^{a}$ TERESA LLUCH-CANUT ${ }^{3}$, ALEJANDRO GARCÍA-LORENZO 4 Y JOAQUÍN TOMÁS-SÁBADO²}

${ }^{1}$ Escola Universitària d'Infermeria Gimbernat. Barcelona.

2Unitat de Recerca i Comunicació Científica (URICC). Escoles Universitàries Gimbernat. Barcelona.

${ }^{3}$ Escuela de Enfermería. Universitat de Barcelona.

${ }^{4}$ Hospital Sant Rafael, Germanes Hospitalàries. Barcelona.

\section{RESUMEN}

Introducción: el estigma de la enfermedad mental es un fenómeno ampliamente arraigado en nuestra sociedad. El desarrollo de programas eficaces para la erradicación del estigma necesita disponer de instrumentos dedicados a evaluar las intervenciones diseñadas. El objetivo del presente trabajo es adaptar y validar la versión española de la escala CAMI-S (Community Attitudes towards Mental Illness, Swedish version) en una muestra de estudiantes de enfermería.

Material y método: la escala original en inglés se tradujo al español mediante sucesivos ciclos de traducción y retrotraducción. El estudio se ajustó a un diseño observacional transversal, correlacional. Los participantes fueron 273 estudiantes de Grado de Enfermería de la Escuela Universitaria de Enfermería Gimbernat, de Sant Cugat del Vallès (Barcelona).

Resultados: se obtuvo un coeficiente alfa de Cronbach de 0,790 para la escala CAMI-S y una estructura factorial coherente y significativa, formada por tres factores, al igual que el instrumento original.

Conclusiones: los resultados apoyan la consideración de la escala CAMI-S como un instrumento válido y fiable para la evaluación del estigma en la población española.

Palabras clave: estigma, salud mental, enfermería.

Correspondencia: Meritxell Sastre-Rus

Correo electrónico: meritxell.sastre@eug.es 


\section{INTRODUCCIÓN}

El estigma de la enfermedad mental es un fenómeno ampliamente arraigado en nuestra sociedad, con importantes consecuencias negativas para la persona que sufre un trastorno mental. El diseño de estrategias para eliminar — 0 , al menos, disminuir — este estigma constituye un objetivo prioritario de los programas desarrollados a nivel internacional ${ }^{1}$.

El concepto de estigma también comporta en su definición otros componentes añadidos, como la peligrosidad percibida, la distancia social, los estereotipos, los prejuicios y la discriminación². El estigma causa perjuicios a la persona que lo padece, como dificultad para el acceso al empleo remunerado, a los servicios de salud o a la vivienda, o dificultad en las relaciones y en las oportunidades educativas. Además, la sociedad reacciona con miedo al estigma y aumenta las distancias con la persona estigmatizada ${ }^{3}$.

Las personas con un trastorno mental han experimentado prejuicio y discriminación a lo largo de los años, siendo el fenómeno del estigma público el más estudiado por los investigadores por las consecuencias que este provoca en la sociedad. El autoestigma también causa graves dificultades para la adaptación de la persona a la sociedad, y ambos estigmas aplicados a la salud mental producen disrupciones en la adaptación social y consecuencias negativas en la recuperación de la persona portadora. Estos efectos han promovido la inversión de métodos a nivel individual y colectivo para la reducción del estigma en la salud mental ${ }^{4}$. Comprender mejor el estigma de la enfermedad mental y elaborar mecanismos para reducirlo es esencial para mejorar el impacto en la sociedad de las enfermedades mentales 5 .

El estigma implica a los profesionales sanitarios $y$, de manera más significativa, a aquellos que desarrollan su actividad profesional en el entorno de la salud mental. A pesar de las intervenciones existentes dirigidas a los profesionales de la salud men- tal para eliminar el estigma, y el diseño de nuevas medidas para mejorar la imagen social de este grupo de profesionales, siguen persistiendo las actitudes estigmatizantes relacionadas con el colectivo6.

Los profesionales de enfermería son parte de los profesionales del sistema de salud y parte de la sociedad, siendo, por ello, también agentes potencialmente estigmatizadores de la salud mental. Aunque, por su contacto más cercano con la persona enferma, se han identificado con conductas más positivas hacia la salud mental que el resto del grupo de profesionales, continúan manteniendo actitudes paternalistas y sigue existiendo el estigma, especialmente relacionado con la impredecibilidad de las personas con enfermedad mental, así como sus limitadas posibilidades de recuperación? ${ }^{7}$.

El desarrollo de programas eficaces para la erradicación del estigma necesita disponer de instrumentos dedicados a evaluar las intervenciones diseñadas ${ }^{7,8}$. En consecuencia, es necesaria la construcción y adaptación de instrumentos psicométricos válidos y fiables.

A partir de la revisión bibliográfica, se detectaron diversas escalas validadas para la evaluación del estigma, aunque muy pocas dirigidas a profesionales sanitarios y ninguna elaborada para los profesionales de enfermería. En la década de 1980, Taylor y Dear ${ }^{9}$ elaboraron la escala CAMI (Community Attitudes towards the Mentally III), con la intención de proporcionar un instrumento útil para predecir y explicar las reacciones de la comunidad hacia las personas con trastorno mental grave. El cuestionario CAMI, en su versión de 40 ítems, consta de cuatro factores originalmente denominados autoritarismo, benevolencia, restricciones sociales e ideología comunitaria sobre la salud mental, con validez y fiabilidad satisfactorias. Este cuestionario ha sido traducido y adaptado a numerosos idiomas, entre ellos, el sueco ${ }^{10}$, el portugués ${ }^{11}$, el italiano ${ }^{12}$, el chino $^{13} \mathrm{y}$ el eslovaco ${ }^{14}$

En un estudio realizado por Högberg et al. ${ }^{10}$, se eliminaron aquellos ítems que mostraban una pobre 
correlación, quedando la escala reducida a 20 ítems, que fue denominada CAMI-S (en la que la «S» corresponde a Swedish version). Desde esta perspectiva, en este trabajo, se plantea la traducción y adaptación al español de la escala CAMI-S y la determinación de sus propiedades psicométricas, utilizando una muestra de profesionales de enfermería.

\section{MATERIAL Y MÉTODO}

\section{Diseño del estudio}

En este trabajo, se utilizó metodología cuantitativa, aplicando un diseño observacional, transversal, correlacional.

\section{Población de estudio y muestra}

Los participantes en el estudio fueron 273 estudiantes de los cuatro cursos de Grado de Enfermería de la Escuela Universitaria de Enfermería Gimbernat, de Sant Cugat del Vallès (Barcelona). El tamaño muestral se consideró siguiendo las recomendaciones que sugieren utilizar entre cinco y diez sujetos por cada ítem de la escala que se somete a validación ${ }^{15}$. La muestra fue seleccionada siguiendo el criterio de conveniencia. Los criterios de inclusión fueron que se tratase de estudiantes de Grado de Enfermería matriculados en el centro donde se llevó a cabo el estudio, que asistieran el día que se administró el cuestionario y aceptasen participar en el estudio. Los alumnos pertenecían a los cuatro cursos del Grado de Enfermería y respondieron a la escala durante el mes de diciembre de 2016. Todos los sujetos participaron en el estudio de forma voluntaria y se les garantizó el anonimato y la confidencialidad.

En cada ejemplar del cuestionario, se informaba a los participantes sobre la finalidad y la autoría de la investigación, la voluntariedad, el anonimato y la confidencialidad de los datos, así como de la posibilidad de entregar el cuestionario en blan- co si no deseaban participar. Se obtuvo una tasa de respuesta del $98 \%$ de todos los cuestionarios administrados.

\section{INSTRUMENTOS}

Los participantes respondieron un cuestionario anónimo y autoadministrado que contenía, además de datos demográficos, como edad, sexo y curso, las formas españolas de los instrumentos de medida que se detallan en los siguientes apartados.

\section{Escala Community Attitudes towards Mental IIIness (CAMI-S)}

La versión de 20 ítems utilizada en este trabajo ${ }^{10}$ tiene tres factores originalmente etiquetados como mentalidad abierta y favorable a la integración, miedo y evitación, e ideología de la salud mental comunitaria. Los ítems presentan un formato de respuesta de tipo Likert de 5 puntos, siendo: $1=$ total desacuerdo; 2 = considerable desacuerdo; 3 = neutro (ni acuerdo ni desacuerdo); 4 = considerable acuerdo, y $5=$ total acuerdo. Las posibles puntuaciones totales de la escala presentan un intervalo de 20 a 100 puntos, indicando las puntuaciones más altas reacciones más favorables a la integración.

\section{Traducción y adaptación de la escala}

La escala original en inglés se tradujo al español mediante sucesivos ciclos de traducción y retrotraducción. En el proceso, participaron tres personas perfectamente bilingües, que, además, eran expertas en salud mental y psicometría. En primer lugar, los ítems en inglés fueron traducidos al español. Posteriormente, esta traducción fue de nuevo traducida al inglés y comparada con la versión original. Por último, se procedió a una discusión grupal hasta llegar a un consenso, generando, así, la versión final del cuestionario que se utilizaría. 


\section{Escala de Actitud hacia la Inmigración para enfermería (EAl)}

La EAI ${ }^{16}$ es un cuestionario desarrollado para evaluar las actitudes ante el fenómeno de la inmigración en profesionales de enfermería. Consta de 39 ítems con formato de respuesta de tipo Likert de 4 puntos, desde 1 (total acuerdo), hasta 4 (total desacuerdo). De los 39 ítems, 24 de ellos puntúan en sentido negativo, y 15, en sentido positivo. La escala presenta un intervalo de posibles puntuaciones totales de 39 a 156, indicando las puntuaciones más altas actitudes más positivas. En el presente estudio, se obtuvo un coeficiente alfa de consistencia interna para la EAl de 0,951.

\section{Escala de Felicidad de Oxford, Versión Reducida (Oxford Happiness Questionnaire, Short Form o OHQ-SF)}

La OHQ-SF ${ }^{17,18}$ evalúa el bienestar percibido mediante ocho ítems con formato de respuesta de tipo Likert de 6 puntos, de 1 (total desacuerdo) a 6 (total acuerdo), con un intervalo de posibles puntuaciones totales de 8 a 48, donde las puntuaciones más altas indican una percepción de mayor bienestar. La OHQ-SF obtuvo en este estudio un alfa de 0,760.

\section{Escala de Autoestima de Rosenberg (Rosenberg Self-Esteem Scale o RSES)}

La RSES ${ }^{19,20}$ consta de 10 ítems con un formato de respuesta de tipo Likert de 4 puntos, desde total desacuerdo a total acuerdo. Cinco de los ítems puntúan en sentido positivo, de 1 para el total desacuerdo a 4 para el total acuerdo (ítems 1, 3, 4, 7 y 10), mientras que los otros cinco ítems puntúan en sentido negativo, de 4 para el total desacuerdo a 1 para el total cuerdo (ítems 2, 5, 6, 8 y 9), de manera que las posibles puntuaciones totales presentan un intervalo de 10 a 40, siendo la puntuación de 10 la de más baja autoestima y la de 40 la de la más alta. En este estudio, se alcanzó un alfa de Cronbach para la RSES de 0,861.

\section{Escala de Actitud ante el Sida en Enfermería (EASE)}

La EASE ${ }^{21-23}$ es una escala que evalúa las actitudes ante el sida en profesionales de enfermería. Consta de 21 ítems con formato de respuesta de tipo Likert de 5 puntos. La puntuación asignada a cada ítem, en función de su direccionalidad, es de 5 a 1 ( 5 para el total acuerdo y 1 para el total desacuerdo) para los ítems $3,5,7,8,11,14,15$ y 21 ; y de 1 a 5 (1 para el total acuerdo y 5 para el total desacuerdo) para los ítems 1, 2, 4, 6, 9, 10, 12, 13, 16, 17, 18, 19 y 20. Los dos extremos de puntuación de la escala están constituidos por una puntuación máxima de 105, que indica las actitudes más positivas, y una puntuación mínima de 21, que indicará las actitudes más negativas y prejuiciosas. El coeficiente alfa para la EASE en este estudio fue de 0,794.

\section{PROCEDIMIENTO}

\section{Prueba piloto}

Antes de proceder a administrar el cuestionario a la muestra de validación, se realizó una prueba piloto con 15 estudiantes de enfermería, con el objetivo de estudiar la adecuación y comprensión de los ítems. Además del cuestionario, se les facilitó una hoja para evaluar cuestiones relativas a la aplicabilidad del cuestionario, como su extensión, comprensión, idoneidad y claridad en las instrucciones. Los resultados revelaron que el tiempo medio de cumplimentación era de 15 minutos, el $86 \%$ de los participantes manifestaron que les parecía correcta la longitud del cuestionario, y el $82 \%$ indicaron que los enunciados de los ítems eran claros e idóneos para su finalidad. Se modificaron algunos datos referidos al espacio para los datos sociodemográficos. 


\section{ANÁLISIS DE LOS DATOS}

Los datos fueron tabulados y analizados mediante el programa estadístico SPSS 24.0 para Windows. Se calcularon índices descriptivos, el coeficiente alfa de consistencia interna de Cronbach y los coeficientes de correlación de Pearson. Asimismo, se realizó un análisis factorial exploratorio mediante el método de componentes principales y rotación ortogonal Varimax.

\section{ASPECTOS ÉTICOS}

El estudio contó con el permiso de los autores de la escala original y con la aprobación del comité de ética de la investigación del centro donde se llevó a cabo el estudio. Se informó de forma verbal y escrita a todos los participantes del carácter voluntario y confidencial del procedimiento y de su finalidad. También se pidió permiso a los profesores que impartían clase en ese período. La realización de los cuestionarios fue en formato papel.

\section{RESULTADOS}

De los 273 cuestionarios administrados, se eliminaron cuatro por error en su cumplimentación, quedando una muestra final de 269 estudiantes, de los cuales, 220 eran mujeres y 49 eran hombres, con una media de edad de 22,49 años [desviación típi$\mathrm{ca}=4,77]$ y un intervalo de 18 a 55 años.

\section{Consistencia interna}

Se obtuvo un coeficiente alfa de Cronbach de 0,790 para la escala CAMI-S.

\section{Estructura factorial}

Tras comprobar la adecuación de los datos mediante la prueba de Kaiser-Meyer-Olkin (KMO $=0,9)$ y el test de esfericidad de Bartlett (nivel de significación $[p]<0,01$ ), se realizó un análisis factorial exploratorio, aplicando el método de análisis de componentes principales con posterior rotación ortogonal Varimax. A partir del examen visual del gráfico de sedimentación, se forzó el análisis a tres factores, que, en su conjunto, explicaban el 47,2\% de la varianza total. Todos los ítems presentaron cargas factoriales superiores a 0,40 en alguno de los factores. El factor 1, etiquetado como integración y contacto, explicó el 31,93\% de la varianza y obtuvo las mayores cargas factoriales en los ítems $15,16,18,17$, 19, 8, 2, 12 y 7 . El factor 2, que explicó el 8,09\% de la varianza, se etiquetó como distancia social, y presentó las mayores cargas en los ítems 3, 4, 5, 6, 1,13 y 14 . El factor 3 , que explicó el 6,99\% de la varianza, se etiquetó como peligrosidad y evitación y obtuvo las mayores cargas en los ítems 4, 10, 9, 11 y 20 . La tabla 1 contiene la estructura factorial rotada trifactorial, con las cargas superiores a 0,40.

\section{Validez concurrente}

La tabla 2 contiene los coeficientes de correlación de Pearson entre la escala CAMI-S y el resto de medidas utilizadas (EAI, EASE, RSES y OHQ-SF). Como puede observarse, la CAMI-S se correlacionó de forma negativa y significativa $(p<0,01)$ con la escala EAI y la escala EASE $(r=-0,409$ y $r=-0,518$, respectivamente). Por otro lado, se observó que la escala CAMI-S se correlacionó también de forma negativa y significativa $(p<0,05)$ con la escala RSES, aunque, en este caso, el coeficiente de correlación presentó un valor considerablemente menor $(r=$ $-0,154)$. Asimismo, la escala CAMI-S se correlacionó de forma positiva, aunque no significativa, con la escala OHQ-SF $(r=0,103 ; p>0,05)$.

\section{CONCLUSIONES Y DISCUSIÓN}

Aunque a nivel internacional existen múltiples instrumentos para evaluar el estigma de la enfermedad mental, ninguno de ellos ha sido diseñado para 


\begin{tabular}{|c|c|c|c|}
\hline & \multicolumn{3}{|c|}{ Componente } \\
\hline & 1 & 2 & 3 \\
\hline CAMI-S 15 & 0,709 & & \\
\hline CAMI-S 16 & 0,651 & & \\
\hline CAMI-S 18 & 0,642 & & \\
\hline CAMI-S 17 & 0,628 & & \\
\hline CAMI-S 19 & 0,604 & & \\
\hline CAMI-S 8 & 0,492 & & \\
\hline CAMI-S 2 & 0,440 & & \\
\hline CAMI-S 12 & 0,425 & & \\
\hline CAMI-S 7 & 0,412 & & \\
\hline CAMI-S 3 & & 0,731 & \\
\hline CAMI-S 4 & & 0,688 & 0,409 \\
\hline CAMI-S 5 & & 0,610 & \\
\hline CAMI-S 6 & & 0,608 & \\
\hline CAMI-S 1 & & 0,570 & \\
\hline CAMI-S 14 & & 0,564 & \\
\hline CAMI-S 13 & & 0,449 & \\
\hline CAMI-S 10 & & & 0,762 \\
\hline CAMI-S 9 & & & 0,746 \\
\hline CAMI-S 11 & & & 0,741 \\
\hline CAMI-S 20 & & & 0,629 \\
\hline
\end{tabular}

CAMI-S: Community Attitudes towards Mental Illness (version de 20 ítems).

su aplicación específica en profesionales de enfermería.

Uno de los instrumentos validados más utilizado en profesionales sanitarios es el Attribution Question- naire $(A Q)^{24}$. En las adaptaciones realizadas 25,26 y en los estudios de validación en diferentes mues$\operatorname{tras}^{27,28}$, el $A Q$ ha mostrado propiedades métricas aceptables, aunque en ninguno de los trabajos ha 
Tabla 2. Coeficientes de correlación de Pearson entre la CAMI-S y el resto de medidas utilizadas (EAI, EASE, RSES, HQ-SF)

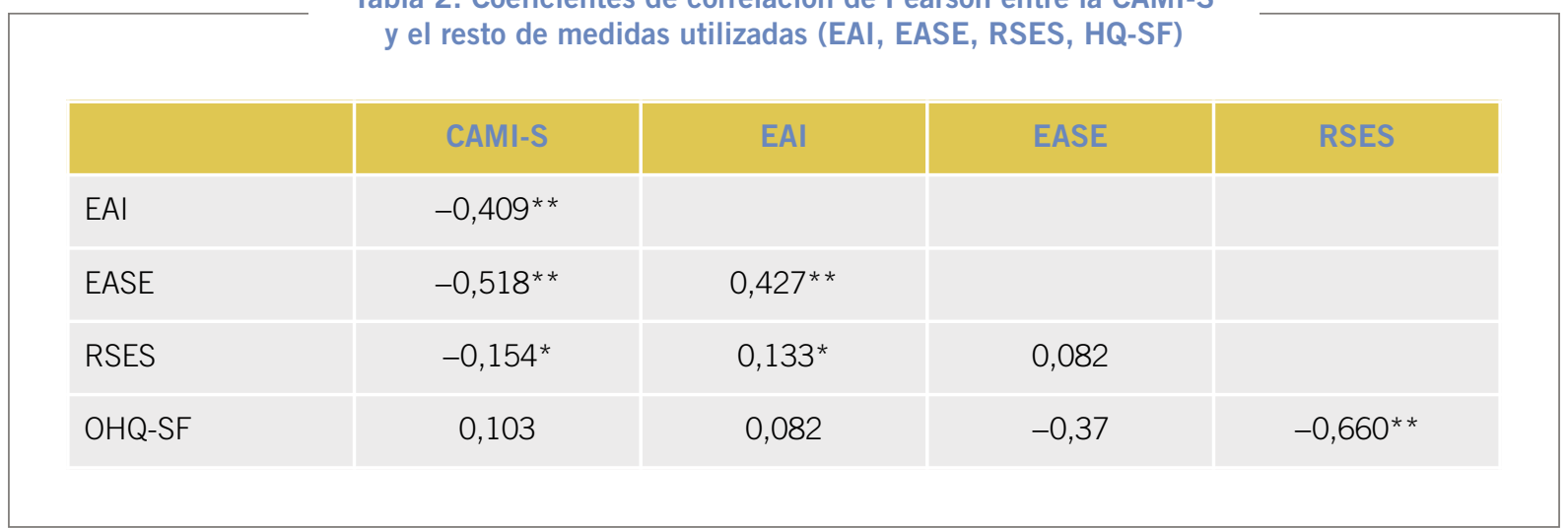

${ }^{* *} p<0,01,{ }^{*} p<0,05$

CAMI-S: Community Attitudes towards Mental Illness (versión de 20 ítems); EAI: Escala de Actitud hacia la Inmigración para enfermería; EASE: Escala de Actitud ante el Sida en Enfermería; OHQ-SF: Escala de Felicidad de Oxford, Versión Reducida (Oxford Happiness Questionnaire, Short Form); RSES: Escala de la Autoestima de Rosenberg (Rosenberg Self-Esteem Scale).

sido aplicado a muestras formadas exclusivamente por profesionales de enfermería. En otro de los instrumentos desarrollados para la medida del estigma de los proveedores de los servicios de salud, el OMS-HC (Opening Minds Scale for Health Care Providers ${ }^{29}$, tampoco se dispone de datos concretos de muestras de enfermeras. Por otra parte, aunque pueden encontrarse otras escalas de medida destinadas a evaluar de forma específica el estigma en los profesionales de la salud menta|30-32, en ningún caso, se aportan estudios que permitan su uso con suficientes garantías de fiabilidad y validez.

Bajo este presupuesto, se planteó la adaptación y validación de la escala CAMI-S en el contexto de profesionales de enfermería españoles. Los resultados obtenidos muestran una adecuada consistencia interna y validez concurrente. Las correlaciones son coherentes con los planteamientos iniciales e indican que existe una relación entre las consideraciones estigmatizadoras hacia el trastorno mental y las actitudes negativas y prejuiciosas hacia el fenómeno de la inmigración (escala EAl) y hacia las personas afectadas por el síndrome de inmunodeficiencia adquirida (escala EASE). Por otro lado, no parece que el bienestar subjetivo (OHQ-SF) sea una varia- ble con influencia destacable sobre las actitudes hacia el trastorno mental.

El análisis factorial exploratorio identificó una estructura factorial coherente y significativa, formada por tres factores, al igual que el instrumento original, con una distribución de cargas de los ítems a los respectivos factores, con similitudes y diferencias con respecto a la obtenida en la validación de la versión inglesa de la CAMI-S. Debido a estas diferencias observadas entre ambas soluciones factoriales, se decidió modificar las denominaciones de los tres factores de la forma española de la escala, que fueron etiquetados como integración y contacto, distancia social y peligrosidad y evitación. Los datos obtenidos en nuestra muestra coinciden con lo ya observado por los autores de la escala CAMI-S en el contexto sueco ${ }^{10}$.

Este estudio presenta algunas limitaciones. Tal vez la más importante de ellas sea la naturaleza de la muestra utilizada para la validación de la CAMI-S, formada exclusivamente por estudiantes de enfermería, algunos de los cuales ya han tenido experiencias con personas con trastorno mental, ya sea en sus prácticas clínicas o bien a nivel laboral. No obstante, los investigadores son poco unánimes en 
la consideración de la influencia que el contacto previo con estos pacientes pueda tener en la actitud posterior hacia la enfermedad mental. Deberían plantearse futuros estudios dedicados a contrastar nuestros datos con los obtenidos en muestras de profesionales de enfermería, ya que el contacto en el ámbito laboral con personas afectadas por un trastorno mental podría modificar la actitud de estos profesionales, como sugieren algunos estudios previos ${ }^{10}$.

En conclusión, los resultados obtenidos concuerdan, básicamente, con los obtenidos por los autores de la versión original y, por lo tanto, apoyan la consideración de la escala CAMI-S como un instrumento válido y fiable para la evaluación del estigma en la población española, que puede utilizarse en ampliar el ámbito de investigación acerca de las reacciones de la comunidad a los servicios que atienden las necesidades de las personas con un trastorno mental.

\section{BIBLIOGRAFÍA}

1. Organización Mundial de la Salud (OMS). La salud mental y los adultos mayores. Ginebra: OMS; 2016. Disponible en: http://www.who.int/mediacentre/facts heets/fs381/es/

2. Zartaloudi A, Madianos M. Stigma related to help-seeking from a mental health professional. Health Sci J. 2010;2(4):77-83.

3. Corrigan P, Larson J. Stigma. En: Mueser KT, Jeste K (eds.). Clinical handbook of schizophrenia. Nueva York: Guilford Press; 2008. p. 533-40.

4. Corrigan PW, Rao D. On the self-stigma of mental illness: stages, disclosure, and strategies for change. Can J Psychiatry. 2012;57(8):464-9.

5. Watson A, Corrigan P, Kosyluk K. Challenging stigma. En: Byrne P, Rosen A (eds.). Early intervention in psychiatry: El of nearly everything for better mental health. Londres: Wiley-Blackwell; 2014. p. 358-72.

6. Gaebel W, Zäske H, Zielasek J, Cleveland HR, Samjeske K, Stuart H, et al. Stigmatization of psychiatrists and general practitioners: results of an international survey. Eur Arch Psychiatry Clin Neurosci. 2015; 265(3):189-97.

7. Corrigan PW, Powell KJ, Michaels PJ. Brief battery for measurement of stigmatizing versus affirming attitudes about mental illness. Psychiatry Res. 2014;215(2):46670 .
8. Ungar T, Knaak S, Szeto AC. Theoretical and practical considerations for combating mental illness stigma in health care. Community Ment Health J. 2016;52(3): 262-71.

9. Taylor SM, Dear MJ. Scaling community attitudes toward the mentally ill. Schizophr Bull. 1981;7(2):225-40.

10. Högberg T, Magnusson A, Ewertzon M, Lützén K. Attitudes towards mental illness in Sweden: adaptation and development of the Community Attitudes towards Mental Illness questionnaire. Int J Ment Health Nurs. 2008; 17(5):302-10.

11. Siqueira SR, Abelha L, Lovisi GM, Sarução KR, Yang L. Attitudes Towards the Mentally III: a study with health workers at a university hospital in Rio de Janeiro. Psychiatr Q. 2017;88(1):25-38.

12. Buizza C, Pioli R, Ponteri M, Vittorielli M, Corradi A, Minicuci N,et al. Community attitudes towards mental illness and socio-demographic characteristics: an Italian study. Epidemiol Psichiatr Soc. 2005;14(3):15462.

13. Song LY, Chang LY, Shih CY, Lin CY, Yang MJ. Community attitudes towards the mentally ill: the results of a national survey of the Taiwanese population. Int J Soc Psychiatry. 2005;51(2):162-76.

14. Letovancová K, Koval íková N, Dobríková P. Attitude of society towards people with mental illness: the result of national survey of the Slovak population. Int J Soc Psychiatry. 2017;63(3):255-60.

15. Streiner DL. Starting at the beginning: an introduction to coefficient alpha and internal consistency. J Pers Assess. 2003;80(1):99-103.

16. Antonín M, Tomás-Sábado J. La escala de actitud ante la inmigración para enfermería: validación preliminar. Enferm Cient. 2004;(262-263):77-82.

17. Argyle M, Martin M, Lu L. Testing for stress and happiness: the role of social and cognitive factors. En: Spielberg CD, Sarason IG, Brebner MT, Greenglass E, Laungani $P$, O'Roark AM (eds.). Series in stress and emotion: anxiety, anger, and vuriosity, Vol. 15. Stress and emotion: anxiety, anger, and curiosity. Filadelfia: Taylor \& Francis; 1995. p. 173-87.

18. Hills P, Argyle M. The Oxford Happiness Questionnaire: a compact scale for the measurement of psychological well-being. Pers Individ Dif. 2002;33(7):1073-82.

19. Rosenberg M. Society and the adolescent self-image. Princeton: Princeton University Press; 1965.

20. Tomás-Sábado J, Limonero JT. Propiedades psicométricas de la Escala de Autoestima de Rosemberg (RSES) en una muestra española. [Monografía en internet]. Interpsiquis; 2008.

21. Tomás-Sábado J. Actitud de enfermería ante el sida. Construcción de una escala de Likert. Enf Clin. 1999; 9(6):233-7. 
22. Tomás-Sábado J, Aradilla A. Actitud ante el sida en estudiantes de enfermería. ¿Cuál es el papel de la formación académica? Educ Med. 2003;6(2):87-92.

23. Fernández L, Fernández $P$, Tomás-Sábado J. Modificación de actitudes ante el Sida en estudiantes de enfermería. Resultados de una experiencia pedagógica. Educ Med. 2006;9(2):84-90.

24. Corrigan P, Markowitz FE, Watson A, Rowan D, Kubiak MA. An attribution model of public discrimination towards persons with mental illness. J Health Soc Behav. 2003;44(2):162-79.

25. de Araujo A, Elói SM, Delbone RM. Brazilian version of the Attribution Questionnaire - Cross cultural adaptation and validation of psychometric properties. J Bras Psiquiatr. 2016;65(4):314-21.

26. Pingani L, Forghieri M, Ferrari S, Ben-Zeev D, Artoni P, Mazzi F, et al. Stigma and discrimination toward mental illness: translation and validation of the Italian version of the Attribution Questionnaire-27 (AQ-27-I). Soc Psychiatry Psychiatr Epidemiol. 2012;47(6):993-9.

27. Pingani L, Catellani S, Del Vecchio V, Sampogna G, Ellefson SE, Rigatelli M, et al. Stigma in the context of schools: analysis of the phenomenon of stigma in a population of university students. BMC Psychiatry. 2016;16:29.

28. Corrigan PW, Powell KJ, Michaels PJ. Brief battery for measurement of stigmatizing versus affirming attitudes about mental illness. Psychiatry Res. 2014;215(2): 466-70.

29. Kassam A, Papish A, Modgill G, Patten S. The development and psychometric properties of a new scale to measure mental illness related stigma by health care providers: the Opening Minds Scale for Health Care Providers (OMS-HC). BMC Psychiatry. 2012;12(1):6274.

30. Chiles C, Stefanovics E, Rosenheck R. Attitudes of students at a US medical school toward mental illness and its causes. Acad Psychiatry. 2017;41(3):320-5.

31. Fuemeaier A, Tucha L, Koerts J, Mueller AK, Lange $\mathrm{KW}$, Tucha O. Measurement of stigmatization towards adults with attention deficit hyperactivity disorder. Plos One. 2012;7(12):e51755.

32. Tei-Tominaga M, Asakura T, Asakura K. Stigma towards nurses with mental illnesses: a study of nurses and nurse managers in hospitals in Japan. Int J Ment Health Nurs. 2014;23(4):316-25. 